\title{
STUDIES ON THE CHEMICAL COMPONENTS OF MORINGA OLEIFERA PLANT GROWN UNDER EGYPTIAN CONDITIONS
}

\author{
E.A.E. El-Ghadban ${ }^{*}$; G.F. Mahmud ${ }^{* *}$ and C.H. Carrie ${ }^{* *}$ \\ * Medicinal and Aromatic Plants Res. Dept., Hort. Res. Inst. \& National Gene Bank, ARC, Giza, Egypt. \\ ** Functional Foods Dept., National Inst. of Nutrition Canada, Ottawa.
}

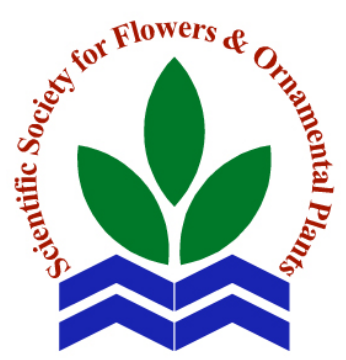

Scientific J. Flowers \& Ornamental Plants, 2(1):101-115 (2015).

Received:

$11 / 12 / 2014$

Revised by:

Prof. Dr. E.S. Nofal, Kafr El-Sheikh Univ.

Prof. Dr. A.Z. Sarhan, Cairo Univ.

ABSTRACT: This study was conducted in Department of Medicinal and Aromatic Plants, Horticultural Research Institute, ARC, Dokki, in two successive seasons of 2012 and 2013 on Moringa oleifera plant in 4 (four) different regions in the Upper Egypt (A1 and A2) and Delta (A3 and A4). It aimed to study the effect of different environments on chemicals and food ingredients, as well as determine the adaptation to different environments to identify the best region for growth. The seeds were germinated in the greenhouse of National Gene Bank, and then seedlings were transferred into (A1, A2, A3 and A4 regions) in plots with $20 \times 30 \mathrm{~m}$ distance with $2 \mathrm{~m}$ distance between the plant and $3 \mathrm{~m}$ distance between rows. Samples were taken in different growth stages $(2,4,6,8,10$ and 12 month).

The obtained results showed that there were significant differences in chemical components in the whole regions under study. The region (A1) showed the best values in respect to acid ascorbic (vitamin C), beta carotene (vitamin A), thiamine (vitamin B1), riboflavin (vitamin B2), niacin (vitamin B3), and tocopherols (vitamin E), calcium, iron, protein, zinc and phosphorus at a rate value higher than the region (A2) by $2 \%$, region (A3) by $15 \%$ and region (A4) by $8 \%$. The obtained results explained that the differences among regions in chemical and food components during plant growth. The best results were obtained from the Upper Egypt under study in respect to the quantity of nutrition in addition to region (A4) which were good for cultivating of Moringa tree in Egypt.

Key words: Moringa oleifera, Moringa trees, Nutritional Value of Moringa, Moringa oleifera medicinal properties.

\section{INTRODUCTION}

The Moringa oleifera genus is a common member of the family Moringaceae, which containing a wide range of plants, including flowering herbs and trees. It is common known as Horseradish tree, Benzolive, Kelor or Drumstick tree. The drumstick-like shape, curved seed pods is the characteristic for this species calling Drumstick tree (Asres, 1995). This tree is origin in Himalayas, India and it grows in tropical and semi-arid climates. Moringa tree reaches to about ten meters in height and be drought tolerant allowing it to thrive in arid climates. In addition, the Moringa trees has multi-uses as a food and medicinal plant (Costa-Lotufo et al., 2005), and each part of tree utilized to benefit humans, provided food, and other valuable materials for farming and fuel (Dahot, 1998).

The pods and leaves of Moringa trees was used for food in numerous cultures throughout the world. According to Bharali et al. (2003), it cultivated first in Northern India and incorporated into a number of 


\section{E.A.E. El Ghadban et al.}

religious and cultural observances. Derivedoils from the seeds used as food and in unguents by the ancient Greeks, Romans and Egyptians and were part of the Ayurvedic health diet in India (Badgett, 1964; Anderson et al., 1986). Oil products, valuable food source, changing in environment and culture and increasing interest of Moringa uses in over the world has led to its cultivation in many regions as well as the West India. The leaves, flowers and pods have highly nutritional and provide a number of necessary nutrients, including protein, betacarotene, calcium and vitamin C (Bharali et al., 2003). Because of it made spread in a wide range of climates in order to produce the different parts of plant for utilizing in more uses. It is well good to use as a nutrition (either humans or animals) in the poor regions in over the world, including Asia and Africa, making it more useful for fighting malnutrition in these regions (D'Souza and Kulkarni, 1993).

The shelf life of food is the biggest one advantage in Moringa tree for malnutritional fighting. The leaves of Moringa are consumed fresh, dried or cooked as spinach, in addition to store for months without requiring refrigeration (Caceres and Lopez, 1991; Caceres et al., 1991 and 1992). The fresh leaves are a source of vitamin $\mathrm{C}$, while the dried leaf powder has Vitamin A equal about ten times available than fresh carrot, according to Akhtar and Ahmad (1995) and Anwar and Bhanger (2003). Moreover, the plant has other vitamins and minerals, including high concentrations of calcium, iron, potassium, magnesium and the vitamin B (Babu, 2000; Barminas et al., 1998 and Chawla et al., 1988). The Moringa plant is utilized as a supplemental nutrition specially vitamin and mineral supplements (Bharali et al., 2003).

This study was carried out to study the effect of different environments on plant growth, chemical and food ingredients at different plant growth stages through cultivating in four Egyptian regions.

\section{MATERIALS AND METHODS}

This investigation was carried out at the Experimental Farm of National Gene Bank, and Medicinal and Aromatic Plant Research Department, Horticulture Research Institute, Agricultural Research Center, Dokki.

\section{Seeds used in the experiment:}

Moringa (Moringa oleifera) was planted in four regions in Egypt, in order to study the effect of different environments on the food ingredients and chemicals, as well as determine the significance of this plant and the extent to adapt it to the different environments in Egypt and determine the best Egyptian conditions for growth.

\section{Place of agriculture:}

Seeds of Moringa were planted in two successive seasons of 2012 and 2013 in the four Egyptian regions as the following in: (1) Upper Egypt: (Al-Saf) Giza Governorate $\left(\mathrm{A}_{1}\right)$, and (Beni Mazar) Minya Governorate $\left(\mathrm{A}_{2}\right)$ and (2) Delta area of Egypt: (Nubaria) Beheira Governorate $\left(\mathrm{A}_{3}\right)$ and (Sarabum) Ismailia Governorate $\left(\mathrm{A}_{4}\right)$.

\section{Characteristics of soil:}

In the Upper Egypt $\left(A_{1}, A_{2}\right)$ soil was characteristic as light clay, good drainage and irrigated with water from the Nile River, while in the area North of Egypt $\left(\mathrm{A}_{3}, \mathrm{~A}_{4}\right)$ soil was a good sandy, high penetrating and irrigated with groundwater.

\section{Seedlings of plants in the field experiment:}

Moringa was germinated in the Greenhouse of National Gene Bank before transfer the seedlings to four regions $\left(\mathrm{A}_{1}, \mathrm{~A}_{2}\right.$, $A_{3}$ and $A_{4}$ ) under study. After seedling, the planted plots were $20 \times 30 \mathrm{~m}$; with $2 \mathrm{~m}$ distance between plants and $3 \mathrm{~m}$ distance between the rows. It applied in both seasons (2012 and 2013) in all regions under study. This aimed to study the impact of Egyptian conditions (climatic factors and soil conditions) on food ingredients and chemical components for this plant. 


\section{Sampling of Moringa leaf:}

Samples of leaves were taken at different stages of fresh weight of leaves after seedling $(2,4,6,8,10$ and 12 months in both successive seasons), in order to study the chemical and food ingredients and stages of their formation and quantity through the stages of plant growth under Egyptian conditions in different regions under study.

\section{Sampling of Moringa seeds and roots:}

At the end of growth in two successive seasons of 2012 and 2013, seed samples was be taken from mature pods as well as samples of roots for this plant.

\section{Plant samples analysis:}

Moringa samples were analyzed at the National Institute of Nutrition Canada, Ottawa, Canada.

\section{Chemical characterization of Moringa oleifera:}

Moringa seeds were analyzed for various quality attributes including proximate analysis, mineral composition, polyphenols and alkaloids. The procedures followed are given:

\section{1- Proximate analysis:}

\section{a. Moisture content:}

Moisture contents of Moringa seeds were estimated by drying the samples in an Air Forced Draft Oven (Model: DO-1-30/02, PCSIR, Canada) at $105 \pm 5^{\circ} \mathrm{C}$ until a constant weight was obtained (A.A.C.C., 2000; Method No. 44-15A).

\section{b. Crude protein:}

Crude protein content was determined by using Kjeldahle Apparatus (Model: D40599, Behr Labor Technik, GmbhGermany) as described in A.A.C.C. (2000) Method No. 46-30.

\section{c. Crude fat:}

Content of crude fat was determined using hexane as a solvent in Soxhlet System (Model: H-2 1045 Extraction Unit, Hoganas,
Sweden) according to the procedure give in A.A.C.C. (2000) Method No. 30-25.

\section{d. Crude fiber:}

Crude fiber was estimated in fat free samples by treating with $1.25 \% \mathrm{H}_{2} \mathrm{SO}_{4}$, left over material was subjected to further treatment with $1.25 \% \mathrm{NaOH}$ solutions. Crude fiber of the samples was determined through Labconco Fibertech (Labconco Corporation Kansas, USA) as per procedure in A.A.C.C. (2000) Method No. 32-10.

\section{e. Nitrogen free extract (NFE):}

NFE was calculated according to the following expression: NFE $\%=100-$ (moisture contents $\%+$ crude protein $\%+$ crude fat $\%+$ crude fiber $\%+$ ash $\%$ )

\section{2- Mineral contents:}

Moringa was analyzed for its mineral profile following A.O.A.C. (2003). Concentrations of calcium (Method 968.08), magnesium (Method 968.08), zinc (Method 991.11), iron (Method 985.01) and phosphorous (Method 965.17) were determined by Atomic Absorption Spectrophotometer (Varian AA240, Australia), while sodium (Method 968.08) and potassium (Method 968.08) were measured through Flame Photometer-410 (Sherwood Scientific Ltd., Cambridge).

\section{a. Polyphenols:}

Total polyphenols were determined using Folin-Ciocalteu method and values were expressed as gallic acid equivalent (Singleton et al., 1999; Akowuah et al., 2005). $20 \mathrm{gm}$ of seeds were slurred in $200 \mathrm{ml}$ of methanol. One $\mathrm{ml}$ of methanolic extract $(10 \mathrm{~g} / \mathrm{l})$ was mixed with $5 \mathrm{ml}$ of FolinCiocalteu reagent $(10 \%)$ and $4 \mathrm{ml}$ of sodium carbonate solution $(75 \mathrm{~g} / \mathrm{l})$ and after $30 \mathrm{~min}$ absorbance $(765 \mathrm{~nm})$ was noted on UV/VIS light spectrophotometer (CECIL CE 7200). Calibration/standard curve for gallic acid was drawn with concentrations of $0.05,0.10$, $0.15,0.20,0.25$ and $0.30 \mathrm{mg} / \mathrm{ml}$ methanol mixed with the same reagents. Total polyphenols content was calculated by the following formula: 


\section{E.A.E. El Ghadban et al.}

$\mathrm{C}=\mathrm{c} \times \mathrm{V} / \mathrm{m}$

$\mathrm{C}=$ total content of phenolic compounds in $\mathrm{mg} / \mathrm{g}$ plant extract, in GAE.

$\mathrm{c}=$ the concentration of gallic acid calculated from the calibration curve in $\mathrm{mg} / \mathrm{ml}$.

$\mathrm{V}=$ the volume of extract in $\mathrm{ml}$.

$\mathrm{m}=$ the weight of plant methanolic extract in g.

\section{b. Alkaloids:}

Total alkaloids were determined by following the Method No. 20.20 as described in A.A.C.C. (2000).

\section{Extraction of fixed oil:}

The oil from the Moringa seed was extracted through solvent extraction technique as described in A.O.C.S. (1998); Rotary Evaporator (Eyela, Japan) recovered hexane used as solvent. The extracted oil was stored in dark place at room temperature. Moringa oleifera fixed oil was analyzed for physical \& chemical characteristics and fatty acid profile using their respective methodologies as presented below Acid value Acid value is defined as the milligrams of $\mathrm{KOH}$ required for neutralization of free fatty acids present in one gram of oil. Neutral alcohol was added to fixed oil of Moringa oleifera sample and titrated against $\mathrm{KOH}$ solution according to A.O.C.S. (1998); Method No. Cd 3d-63.

\section{Antioxidant potential of fixed oil:}

\section{Antioxidant activity:}

Antioxidant activity based on coupled oxidation of $\beta$-carotene and linoleic acid were evaluated by using the method described by Taga et al. (1984). Oxidation of $\beta$-carotene emulsion was spectrophotometric monitored by measuring absorbance at 470 $\mathrm{nm}$ after $0,10,20,30$ and $40 \mathrm{~min}$. The degradation rate of the extracts was calculated according to first order kinetics using following equation (Al-Saikhan et al., 1995).

$$
\text { In }(a / b) \times 1 / t=\text { sample degradation rate }
$$

In $=$ the natural $\log$.

$\mathrm{a}=$ the initial absorbance $(470 \mathrm{~nm})$ at time zero.

$\mathrm{b}=$ the absorbance $(470 \mathrm{~nm})$ after $40 \mathrm{~min}$.

$\mathrm{t}=$ the time $(\mathrm{min})$.

The antioxidant activity (AA) was expressed as \% inhibition relative to the control using following equation its use in the study plan

\section{RESULTS AND DISCUSSION}

Data presented in Table (1) show the nutrient values after two months in Moringa plant in the conditions of four Egyptian regions in two successive seasons of 2012 and. The obtained data showed that vit A values ranged from 5.1935 to $6.11 \mathrm{mg}$. A deficiency of vit. A may be caused blindness each year especially in the developing countries may reach approximately 250,000 to 500,000 malnourished children (Abrams et al., 1993 and Abuye et al., 1999). In 2002, the set of United Nations Special Session on children reported that the elimination of vitamin A deficiency by 2010 (Wikipedia, 2010). It is unfortunate that over 100 million children around the world may go blind simply because they are not getting enough vit A. A few spoonful in the children's food could easily save them from going blind (Wikipedia, 2010).

From Table (1), the values of vit. C ranged from 186.167 to $219.022 \mathrm{mg}$. The values of calcium in the experiment ranged from 359.55 to $423 \mathrm{mg}$. Iron in the experiment ranged from 0.679 to $0.799 \mathrm{mg}$. The values of niacin (vit. $\mathrm{B}_{3}$ ) ranged from 0.639 to $0.752 \mathrm{mg}$. The values of protein ranged from 5433.2 to $6392 \mathrm{mg}$. The values of tocopherols in the experiment ranged from 23.171 to $27.26 \mathrm{mg}$. Zinc in the experiment ranged from 0.14382 to $0.169 \mathrm{mg}$. vitamin $\mathrm{B}_{2}$ (riboflavin) ranged from 0.0399 to 0.047 $\mathrm{mg}$. The values of thiamin (vit. $\mathrm{B}_{1}$ ) ranged from 0.479 to 0.564 . The obtained data from Table (1) showed region $\left(A_{1}\right)$ representing Al-Saf, Giza Governorate are higher in nutrients and chemical components while the 
Table 1. Analysis of the components of Moringa cultivated under Egyptian conditions after 2 months of agriculture.

\begin{tabular}{|c|c|c|c|c|c|c|c|c|}
\hline \multirow{2}{*}{ Compound } & \multicolumn{4}{|c|}{2012} & \multicolumn{4}{|c|}{2013} \\
\hline & $\mathbf{A}_{1}$ & $\mathbf{A}_{2}$ & $\mathbf{A}_{3}$ & $\mathbf{A}_{4}$ & $\mathbf{A}_{1}$ & $\mathbf{A}_{2}$ & $\mathbf{A}_{3}$ & $\mathbf{A}_{4}$ \\
\hline ASCORBIC-ACID * & 219.020 & 214.640 & 186.167 & 201.498 & 219.022 & 214.642 & 186.169 & 201.500 \\
\hline BETA-CAROTENE & 6.110 & 5.988 & 5.194 & 5.621 & 6.112 & 5.990 & 5.196 & 5.623 \\
\hline CAFFEIC-ACID & 1.410 & 1.382 & 1.199 & 1.297 & 1.412 & 1.384 & 1.201 & 1.299 \\
\hline CALCIUM & 423.00 & 414.54 & 359.55 & 389.16 & 423.00 & 414.54 & 359.55 & 389.16 \\
\hline CARBOHYDRATES & 11750 & 11515 & 9987.5 & 10810 & 11750 & 11515 & 9987.5 & 10810 \\
\hline CHOLINE & 282 & 276 & 240 & 259 & 282 & 276 & 240 & 259 \\
\hline COPPER & 0.0658 & 0.0645 & 0.0559 & 0.0605 & 0.0678 & 0.0665 & 0.0579 & 0.0625 \\
\hline FAT & 1598 & 1566 & 1358 & 1470 & 1598 & 1566 & 1358 & 1470 \\
\hline FIBER & 846 & 829 & 719 & 778 & 846 & 829 & 719 & 778 \\
\hline FLUORINE & 1.880 & 1.842 & 1.598 & 1.730 & 1.882 & 1.844 & 1.600 & 1.732 \\
\hline IODINE & 0.470 & 0.461 & 0.400 & 0.432 & 0.472 & 0.463 & 0.402 & 0.434 \\
\hline IRON & 0.799 & 0.783 & 0.679 & 0.735 & 0.801 & 0.785 & 0.681 & 0.737 \\
\hline KAEMPFEROL & 1.880 & 1.842 & 1.598 & 1.730 & 1.882 & 1.844 & 1.600 & 1.732 \\
\hline Magnesium & 0.376 & 0.369 & 0.320 & 0.346 & 0.378 & 0.371 & 0.322 & 0.348 \\
\hline NIAZIMIN & 2.820 & 2.764 & 2.397 & 2.594 & 2.822 & 2.766 & 2.399 & 2.596 \\
\hline Niacin (vitamin B3) & 0.7520 & 0.7370 & 0.6392 & 0.6918 & 0.7540 & 0.7390 & 0.6412 & 0.6938 \\
\hline OXALATE & 0 & 0 & 0 & 0 & 0 & 0 & 0 & 0 \\
\hline OXALIC-ACID & 0 & 0 & 0 & 0 & 0 & 0 & 0 & 0 \\
\hline PHOSPHORUS & 65.800 & 64.484 & 55.930 & 60.536 & 65.802 & 64.486 & 55.932 & 60.538 \\
\hline PROLAMINE & 81.592 & 79.960 & 69.353 & 75.065 & 81.594 & 79.962 & 69.355 & 75.067 \\
\hline PROTEIN & 6392 & 6264 & 5433 & 5880 & 6392 & 6264 & 5433 & 5881 \\
\hline Potassium & 239.70 & 234.91 & 203.75 & 220.52 & 239.70 & 234.91 & 203.75 & 220.51 \\
\hline $\begin{array}{l}\text { RIBOFLAVIN } \\
\text { (vitamin B2) }\end{array}$ & 0.0470 & 0.0461 & 0.0400 & 0.0432 & 0.0490 & 0.0481 & 0.0420 & 0.0452 \\
\hline Zinc & 0.1692 & 0.1658 & 0.1438 & 0.1557 & 0.1712 & 0.1678 & 0.1458 & 0.1577 \\
\hline TOCOPHEROLS & 27.260 & 26.715 & 23.171 & 25.079 & 27.262 & 26.717 & 23.173 & 25.081 \\
\hline Thiamin (vitamin B1) & 0.5640 & 0.5527 & 0.4794 & 0.5189 & 0.5660 & 0.5547 & 0.4814 & 0.5209 \\
\hline
\end{tabular}

All values are per 100 grams of edible portion; * The whole concentration values are mg. 


\section{E.A.E. El Ghadban et al.}

region (A3) representing Nubaria, El-Beheira Governorate gave less values, in respect to the values of nutrients ascorbic acid, betacarotene, calcium, iron, tocopherols, vit. $\mathrm{B}_{1}$, $\mathrm{B}_{2}$ and $\mathrm{B}_{3}$ and phosphorus. Whereas regions A4 (Sarabum, Ismailia Governorate) and A2 (Beni Mazar, El-Minya Governorate) gave the moderate values. The obtained results reveal the significant differences in nutrient components.

The region $\mathrm{A} 1$ was at a rate value higher than the region $\mathrm{A} 2$ by $2 \%$, region $\mathrm{A} 3$ by $15 \%$ and region $\mathrm{A} 4$ by $8 \%$. The obtained results explained the differences among regions in chemical and nutrient components during plant growth. The best results was obtained from Upper Egypt under study in respect to the quantity of nutrients. This may be due to the relative variation of the climate and the nature of the soil and irrigation water in each region. The moral differences through growth in 2012 and 2013 seasons, about +/0.002 .

Table (2) showed the chemical and nutrient components after four months. It was noted the increasing in the nutrient contents in each region after four months than at two months. This explains that the nutrients components may increase the effective life of the plant. The values of these nutrient in Table (1), (219.02, 6.11, 423, $1.88,0.376,0.752,65.8,6392,239.7,0.047$, $0.1692,27.26$ and 0.564 ; respectively); while in Table (2) $(221.35,6.175,427.5,1.90$, $0.38,0.76,66.5,6460,242.25,0.0475$, $0.171,27.55$ and 0.57 ; respectively).

The analysis of chemical and nutrient components after six months are show in Table (3). The values of these nutrients in Table (1), respectively $(219.02,6.11,423$, $1.88,0.376,0.752,65.8,6392,239.7,0.047$ $0.1692,27.26$ and 0.564 ), while in Table (3) (223.68, 624, 432, 1.92, 0.384, 0.748, 67.2, $6528,244.8,0.0480,0.1728,27.84$ and 0.576). It was noted the increasing in the nutrient contents in each region after four months than after two months. This explains that the nutrient components may increase the effective life of the plant.
Table (4) showed the components of chemicals and nutrient after 8 months. The values of these nutrient in Table (1), respectively $(219.02,6.11,423,1.88,0.376$, $0.752,65.8,6392,239.7,0.047,0.1692$, 27.26 and 0.564 ), while in Table (4) (226.01, $6.305,436.5,1.94,0.388,0.776,67.9,6596$, $247.35,0.0485,0.1746,28.13$ and 0.582$)$. It was noted the increasing in the nutrient contents in each region after four months than after two months. This explains that the nutrient components may increase the effective life of the plant.

Table (5) showed the chemical and nutrient components after 10 months of agriculture. The values of these nutrients in Table (1), respectively $(219.02,6.11,423$, $1.88,0.376,0.752,65.8,6392,239.7,0.047$, $0.1692,27.26$ and 0.564$)$, while in Table (5) (228.34, 6.37, 441, 196, 0.392, 0.784, 68.6, $6664,249.9,0.4,049,0.1764,28.42$ and 0.588 ). It was noted the increasing on the nutrient contents in each region after four months than after two months. This explains that the nutrient components may increase the effective life of the plant.

Table (6) showed the analysis of chemical and nutrient components after 12 months of agriculture. The values of these nutrient in Table (1), respectively (219.02, $6.11,423,1.88,0.376,0.752,65.8,6392$, $239.7,0.047,0.1692,27.26$ and 0.564 ), while in Table (3) $(233,6.500,450,2.00$, $0.400,0.800,70,6800,255,0.050,0.180$, 29.00 and 0.600 ). It was noted the increasing in the nutrient contents in each region after four months than after two months. This explains that the nutrient components may increase the effective life of the plant.

Table (7) shows the analysis of chemical components in Moringa roots in four regions under study. The obtained results from show that the highest value of chemical components in roots was in region $A_{1}$, while the lowest value was in region $\mathrm{A}_{3}$.

Table (8) shows the results of the chemical components of Moringa seeds in four places under study. The region $A_{1}$ was 
Table 2. Analysis of the components of Moringa cultivated under Egyptian conditions after 4 months of agriculture.

\begin{tabular}{|c|c|c|c|c|c|c|c|c|}
\hline \multirow{2}{*}{ Compound } & \multicolumn{4}{|c|}{2012} & \multicolumn{4}{|c|}{2013} \\
\hline & $\mathbf{A}_{1}$ & $\mathbf{A}_{\mathbf{2}}$ & $\mathbf{A}_{3}$ & $\mathbf{A}_{4}$ & $\mathbf{A}_{1}$ & $\mathbf{A}_{2}$ & $\mathbf{A}_{3}$ & $\mathbf{A}_{4}$ \\
\hline ASCORBIC-ACID * & 221.350 & 216.923 & 188.148 & 203.642 & 221.352 & 216.925 & 188.150 & 203.644 \\
\hline BETA-CAROTENE & 6.175 & 6.052 & 5.249 & 5.681 & 6.177 & 6.054 & 5.251 & 5.683 \\
\hline CAFFEIC-ACID & 1.425 & 1.397 & 1.211 & 1.311 & 1.427 & 1.399 & 1.213 & 1.313 \\
\hline CALCIUM & 427.50 & 418.95 & 363.38 & 393.30 & 427.502 & 418.952 & 363.377 & 393.302 \\
\hline CARBOHYDRATES & 11875 & 11638 & 10094 & 10925 & 11875 & 11638 & 10094 & 10925 \\
\hline CHOLINE & 285.00 & 279.30 & 242.25 & 262.20 & 285.002 & 279.302 & 242.252 & 262.202 \\
\hline COPPER & 0.0665 & 0.0652 & 0.0565 & 0.0612 & 0.0685 & 0.0672 & 0.0585 & 0.0632 \\
\hline FAT & 1615.0 & 1582.7 & 1372.8 & 1485.8 & 1615.002 & 1582.702 & 1372.752 & 1485.802 \\
\hline FIBER & 855.00 & 837.90 & 726.75 & 786.60 & 855.002 & 837.902 & 726.752 & 786.602 \\
\hline FLUORINE & 1.900 & 1.862 & 1.615 & 1.748 & 1.902 & 1.864 & 1.617 & 1.750 \\
\hline IODINE & 0.4750 & 0.4655 & 0.4038 & 0.4370 & 0.4770 & 0.4675 & 0.4058 & 0.4390 \\
\hline IRON & 0.8075 & 0.7914 & 0.6864 & 0.7429 & 0.8095 & 0.7934 & 0.6884 & 0.7449 \\
\hline KAEMPFEROL & 1.900 & 1.862 & 1.615 & 1.748 & 1.902 & 1.864 & 1.617 & 1.750 \\
\hline Magnesium & 0.3800 & 0.3724 & 0.3230 & 0.3496 & 0.3820 & 0.3744 & 0.3250 & 0.3516 \\
\hline NIAZIMIN & 2.850 & 2.793 & 2.423 & 2.622 & 2.852 & 2.795 & 2.425 & 2.624 \\
\hline Niacin (vitamin B3) & 0.7600 & 0.7448 & 0.6460 & 0.6992 & 0.7620 & 0.7468 & 0.6480 & 0.7012 \\
\hline OXALATE & 0 & 0 & 0 & 0 & 0 & 0 & 0 & 0 \\
\hline OXALIC-ACID & 0 & 0 & 0 & 0 & 0 & 0 & 0 & 0 \\
\hline PHOSPHORUS & 66.500 & 65.170 & 56.525 & 61.180 & 66.502 & 65.172 & 56.527 & 61.182 \\
\hline PROLAMINE & 82.460 & 80.812 & 70.091 & 75.863 & 82.462 & 80.813 & 70.093 & 75.865 \\
\hline PROTEIN & 6460 & 6330.8 & 5491 & 5943.2 & 6460 & 6330.8 & 5491 & 5943.2 \\
\hline Potassium & 242.250 & 237.405 & 205.911 & 222.870 & 242.252 & 237.407 & 205.915 & 222.872 \\
\hline $\begin{array}{l}\text { RIBOFLAVIN } \\
\text { (vitamin B2) }\end{array}$ & 0.0475 & 0.0466 & 0.0404 & 0.0437 & 0.0495 & 0.0486 & 0.0424 & 0.0457 \\
\hline Zinc & 0.1710 & 0.1676 & 0.1454 & 0.1573 & 0.1730 & 0.1696 & 0.1474 & 0.1593 \\
\hline TOCOPHEROLS & 27.550 & 26.999 & 23.418 & 25.346 & 27.552 & 27.001 & 23.450 & 25.348 \\
\hline Thiamin (vitamin B1) & 0.5700 & 0.5586 & 0.4845 & 0.5244 & 0.5720 & 0.5606 & 0.4865 & 0.5264 \\
\hline
\end{tabular}

All values are per 100 grams of edible portion; * The whole concentration values are mg. 
Table 3. Analysis of the components of Moringa cultivated under Egyptian conditions after 6 months of agriculture.

\begin{tabular}{|c|c|c|c|c|c|c|c|c|}
\hline \multirow{2}{*}{ Compound } & \multicolumn{4}{|c|}{2012} & \multicolumn{4}{|c|}{2013} \\
\hline & $\mathbf{A}_{1}$ & $\mathbf{A}_{2}$ & $\mathbf{A}_{3}$ & $\mathbf{A}_{4}$ & $\mathbf{A}_{1}$ & $\mathbf{A}_{2}$ & $\mathbf{A}_{3}$ & $\mathbf{A}_{4}$ \\
\hline ASCORBIC-ACID * & 223.680 & 219.206 & 190.128 & 205.786 & 223.682 & 219.208 & 190.130 & 205.788 \\
\hline BETA-CAROTENE & 6.240 & 6.115 & 5.304 & 5.741 & 6.242 & 6.117 & 5.306 & 5.743 \\
\hline CAFFEIC-ACID & 1.440 & 1.411 & 1.224 & 1.325 & 1.442 & 1.413 & 1.226 & 1.327 \\
\hline CALCIUM & 432 & 423.36 & 367.20 & 397.44 & 432.002 & 423.362 & 367.202 & 397.442 \\
\hline CARBOHYDRATES & 12000 & 11760 & 10200 & 11040 & 12000 & 11760 & 10200 & 11040 \\
\hline CHOLINE & 288 & 282.24 & 244.80 & 264.96 & 288.002 & 282.242 & 244.802 & 264.962 \\
\hline COPPER & 0.0672 & 0.0659 & 0.0571 & 0.0618 & 0.0692 & 0.0679 & 0.0591 & 0.0638 \\
\hline FAT & 1632 & 1599.36 & 1387.20 & 1501.44 & 1632.002 & 1599.362 & 1387.202 & 1501.442 \\
\hline FIBER & 864 & 846.72 & 734.40 & 794.88 & 864.002 & 846.722 & 734.402 & 794.882 \\
\hline FLUORINE & 1.920 & 1.882 & 1.632 & 1.766 & 1.922 & 1.884 & 1.634 & 1.768 \\
\hline IODINE & 0.4800 & 0.4704 & 0.4080 & 0.4416 & 0.4820 & 0.4724 & 0.4100 & 0.4436 \\
\hline IRON & 0.8160 & 0.7997 & 0.6936 & 0.7507 & 0.8180 & 0.8017 & 0.6956 & 0.7527 \\
\hline KAEMPFEROL & 1.920 & 1.882 & 1.632 & 1.766 & 1.922 & 1.884 & 1.634 & 1.768 \\
\hline Magnesium & 0.3840 & 0.3763 & 0.3264 & 0.3533 & 0.3860 & 0.3783 & 0.3284 & 0.3553 \\
\hline NIAZIMIN & 2.880 & 2.822 & 2.448 & 2.650 & 2.882 & 2.824 & 2.450 & 2.652 \\
\hline Niacin (vitamin B3) & 0.7680 & 0.7526 & 0.6528 & 0.7066 & 0.7700 & 0.7546 & 0.6548 & 0.7086 \\
\hline OXALATE & 0 & 0 & 0 & 0 & 0 & 0 & 0 & 0 \\
\hline OXALIC-ACID & 0 & 0 & 0 & 0 & 0 & 0 & 0 & 0 \\
\hline PHOSPHORUS & 67.200 & 65.856 & 57.120 & 61.824 & 67.202 & 65.858 & 57.122 & 61.826 \\
\hline PROLAMINE & 83.328 & 81.661 & 70.829 & 76.662 & 83.330 & 81.663 & 70.831 & 76.664 \\
\hline PROTEIN & 6528 & 6397.44 & 5548.80 & 6005.76 & 6528.002 & 6397.442 & 5548.802 & 6005.762 \\
\hline Potassium & 244.8 & 239.904 & 208.08 & 225.216 & 244.802 & 239.906 & 208.082 & 225.218 \\
\hline $\begin{array}{l}\text { RIBOFLAVIN } \\
\text { (vitamin B2) }\end{array}$ & 0.0480 & 0.0470 & 0.0408 & 0.0442 & 0.0500 & 0.0490 & 0.0428 & 0.0462 \\
\hline Zinc & 0.1728 & 0.1693 & 0.1469 & 0.1590 & 0.1748 & 0.1713 & 0.1489 & 0.1610 \\
\hline TOCOPHEROLS & 27.840 & 27.283 & 23.664 & 25.613 & 27.842 & 27.285 & 23.666 & 25.615 \\
\hline Thiamin (vitamin B1) & 0.5760 & 0.5645 & 0.4896 & 0.5210 & 0.5780 & 0.5665 & 0.4916 & 0.5319 \\
\hline
\end{tabular}

All values are per 100 grams of edible portion; * The whole concentration values are mg. 
Table 4. Analysis of the components of Moringa cultivated under Egyptian conditions after 8 months of agriculture.

\begin{tabular}{|c|c|c|c|c|c|c|c|c|}
\hline \multirow{2}{*}{ Compound } & \multicolumn{4}{|c|}{2012} & \multicolumn{4}{|c|}{2013} \\
\hline & $\mathbf{A}_{1}$ & $\mathbf{A}_{2}$ & $\mathbf{A}_{3}$ & $\mathbf{A}_{4}$ & $\mathbf{A}_{1}$ & $\mathbf{A}_{2}$ & $\mathbf{A}_{3}$ & $\mathbf{A}_{4}$ \\
\hline ASCORBIC-ACID * & 226.010 & 221.490 & 192.109 & 207.929 & 226.012 & 221.492 & 192.111 & 207.931 \\
\hline BETA-CAROTENE & 6.305 & 6.179 & 5.359 & 5.801 & 6.307 & 6.181 & 5.361 & 5.803 \\
\hline CAFFEIC-ACID & 1.455 & 1.426 & 1.237 & 1.339 & 1.457 & 1.428 & 1.239 & 1.341 \\
\hline CALCIUM & 436.500 & 427.770 & 371.025 & 401.580 & 436.502 & 427.772 & 371.027 & 401.582 \\
\hline CARBOHYDRATES & 12125 & 11882.5 & 10306.3 & 11155 & 12125 & 11882.5 & 10306.3 & 11155 \\
\hline CHOLINE & 291.00 & 285.18 & 247.35 & 267.72 & 291.002 & 285.182 & 247.352 & 267.722 \\
\hline COPPER & 0.0679 & 0.0665 & 0.0577 & 0.0625 & 0.0699 & 0.0685 & 0.0597 & 0.0645 \\
\hline FAT & 1649 & 1616.02 & 1401.65 & 1517.08 & 1649.002 & 1616.022 & 1401.652 & 1517.082 \\
\hline FIBER & 873 & 855.54 & 742.05 & 803.16 & 873.002 & 855.542 & 742.052 & 803.162 \\
\hline FLUORINE & 1.940 & 1.901 & 1.649 & 1.785 & 1.942 & 1.903 & 1.651 & 1.787 \\
\hline IODINE & 0.4850 & 0.4753 & 0.4123 & 0.4462 & 0.4870 & 0.4773 & 0.4143 & 0.4482 \\
\hline IRON & 0.8245 & 0.8080 & 0.7008 & 0.7585 & 0.8265 & 0.8100 & 0.7028 & 0.7605 \\
\hline KAEMPFEROL & 1.940 & 1.901 & 1.649 & 1.785 & 1.942 & 1.903 & 1.651 & 1.787 \\
\hline Magnesium & 0.3880 & 0.3802 & 0.3298 & 0.3570 & 0.3900 & 0.3822 & 0.3318 & 0.3590 \\
\hline NIAZIMIN & 2.910 & 2.852 & 2.474 & 2.677 & 2.912 & 2.854 & 2.476 & 2.679 \\
\hline Niacin (vitamin B3) & 0.7760 & 0.7605 & 0.6596 & 0.7139 & 0.7780 & 0.7625 & 0.6616 & 0.7159 \\
\hline OXALATE & 0 & 0 & 0 & 0 & 0 & 0 & 0 & 0 \\
\hline OXALIC-ACID & 0 & 0 & 0 & 0 & 0 & 0 & 0 & 0 \\
\hline PHOSPHORUS & 67.900 & 66.542 & 57.715 & 62.468 & 67.902 & 66.544 & 57.717 & 62.47 \\
\hline PROLAMINE & 84.196 & 82.512 & 71.567 & 77.460 & 84.198 & 82.514 & 71.569 & 77.462 \\
\hline PROTEIN & 6596 & 6464.08 & 5606.60 & 6068.32 & 6596.002 & 6464.082 & 5606.602 & 6068.322 \\
\hline Potassium & 247.350 & 242.403 & 210.248 & 227.562 & 247.352 & 242.405 & 210.250 & 227.564 \\
\hline $\begin{array}{l}\text { RIBOFLAVIN } \\
\text { (vitamin B2) }\end{array}$ & 0.0485 & 0.0475 & 0.0412 & 0.0446 & 0.0505 & 0.0495 & 0.0432 & 0.0466 \\
\hline Zinc & 0.1746 & 0.1711 & 0.1484 & 0.1606 & 0.1766 & 0.1731 & 0.1504 & 0.1626 \\
\hline TOCOPHEROLS & 28.130 & 27.567 & 23.911 & 25.880 & 28.132 & 27.569 & 23.913 & 25.882 \\
\hline Thiamin (vitamin B1) & 0.5820 & 0.5704 & 0.4947 & 0.5354 & 0.5840 & 0.5724 & 0.4967 & 0.5374 \\
\hline
\end{tabular}

All values are per 100 grams of edible portion; * The whole concentration values are mg. 
Table 5. Analysis of the components of Moringa cultivated under Egyptian conditions after 10 months of agriculture.

\begin{tabular}{|c|c|c|c|c|c|c|c|c|}
\hline \multirow{2}{*}{ Compound } & \multicolumn{4}{|c|}{2012} & \multicolumn{4}{|c|}{2013} \\
\hline & $\mathbf{A}_{1}$ & $\mathbf{A}_{2}$ & $\mathbf{A}_{3}$ & $\mathbf{A}_{4}$ & $\mathbf{A}_{1}$ & $\mathbf{A}_{2}$ & $\mathbf{A}_{3}$ & $\mathbf{A}_{4}$ \\
\hline ASCORBIC-ACID * & 228.34 & 223.773 & 194.089 & 210.073 & 228.342 & 223.775 & 194.091 & 210.075 \\
\hline BETA-CAROTENE & 6.370 & 6.243 & 5.415 & 5.860 & 6.372 & 6.245 & 5.417 & 5.862 \\
\hline CAFFEIC-ACID & 1.470 & 1.441 & 1.250 & 1.352 & 1.472 & 1.443 & 1.252 & 1.354 \\
\hline CALCIUM & 441.00 & 432.18 & 374.85 & 405.72 & 441.002 & 432.182 & 374.852 & 405.722 \\
\hline CARBOHYDRATES & 12250 & 12005 & 10413 & 11270 & 12250 & 12005 & 10413 & 11270 \\
\hline CHOLINE & 294.00 & 288.12 & 249.90 & 270.48 & 294.002 & 288.122 & 249.902 & 270.482 \\
\hline COPPER & 0.0686 & 0.0672 & 0.0583 & 0.0631 & 0.0706 & 0.0692 & 0.0603 & 0.0651 \\
\hline FAT & 1666.00 & 1632.68 & 1416.10 & 1532.72 & 1666.002 & 1632.682 & 1416.102 & 1532.722 \\
\hline FIBER & 882.00 & 864.36 & 749.70 & 811.44 & 882.002 & 864.362 & 749.702 & 811.442 \\
\hline FLUORINE & 1.960 & 1.921 & 1.666 & 1.803 & 1.962 & 1.923 & 1.668 & 1.805 \\
\hline IODINE & 0.4900 & 0.4802 & 0.4165 & 0.4508 & 0.4920 & 0.4822 & 0.4185 & 0.4528 \\
\hline IRON & 0.8330 & 0.8163 & 0.7081 & 0.7664 & 0.8350 & 0.8183 & 0.7101 & 0.7684 \\
\hline KAEMPFEROL & 1.960 & 1.921 & 1.666 & 1.803 & 1.962 & 1.923 & 1.668 & 1.805 \\
\hline Magnesium & 0.3920 & 0.3842 & 0.3332 & 0.3606 & 0.3940 & 0.3862 & 0.3352 & 0.3626 \\
\hline NIAZIMIN & 2.940 & 2.881 & 2.499 & 2.705 & 2.942 & 2.883 & 2.501 & 2.707 \\
\hline Niacin (vitamin B3) & 0.7840 & 0.7683 & 0.6664 & 0.7213 & 0.7860 & 0.7703 & 0.6684 & 0.7233 \\
\hline OXALATE & 0 & 0 & 0 & 0 & 0 & 0 & 0 & 0 \\
\hline OXALIC-ACID & 0 & 0 & 0 & 0 & 0 & 0 & 0 & 0 \\
\hline PHOSPHORUS & 68.600 & 67.228 & 58.310 & 63.112 & 68.602 & 67.230 & 58.312 & 63.114 \\
\hline PROLAMINE & 85.064 & 83.363 & 72.304 & 78.259 & 85.066 & 83.365 & 72.306 & 78.261 \\
\hline PROTEIN & 6664.00 & 6530.72 & 5664.40 & 6130.88 & 6664.002 & 6530.722 & 5664.402 & 6130.882 \\
\hline Potassium & 249.90 & 244.902 & 212.415 & 229.908 & 249.902 & 244.904 & 212.417 & 229.91 \\
\hline $\begin{array}{l}\text { RIBOFLAVIN } \\
\text { (vitamin B2) }\end{array}$ & 0.0490 & 0.0480 & 0.0417 & 0.0451 & 0.0510 & 0.0500 & 0.0437 & 0.0471 \\
\hline Zinc & 0.1764 & 0.1729 & 0.1499 & 0.1623 & 0.1784 & 0.1749 & 0.1519 & 0.1643 \\
\hline TOCOPHEROLS & 28.420 & 27.852 & 24.157 & 26.146 & 28.422 & 27.854 & 24.159 & 26.148 \\
\hline Thiamin (vitamin B1) & 0.5880 & 0.5762 & 0.4998 & 0.5410 & 0.5900 & 0.5782 & 0.5018 & 0.5430 \\
\hline
\end{tabular}

All values are per 100 grams of edible portion; * The whole concentration values are mg. 
Table 6. Analysis of the components of Moringa cultivated under Egyptian conditions after 12 months of agriculture.

\begin{tabular}{|c|c|c|c|c|c|c|c|c|}
\hline \multirow{2}{*}{ Compound } & \multicolumn{4}{|c|}{2012} & \multicolumn{4}{|c|}{2013} \\
\hline & $\mathbf{\mathbf { A } _ { 1 }}$ & $\mathbf{\mathbf { A } _ { 2 }}$ & $\mathbf{\mathbf { A } _ { 3 }}$ & $\mathbf{A}_{4}$ & $\mathbf{A}_{1}$ & $\mathbf{\mathbf { A } _ { 2 }}$ & $\mathbf{\mathbf { A } _ { 3 }}$ & $\mathbf{A}_{4}$ \\
\hline ASCORBIC-ACID * & 233.00 & 228.34 & 198.05 & 214.36 & 233.002 & 228.342 & 198.052 & 214.362 \\
\hline BETA-CAROTENE & 6.500 & 6.370 & 5.525 & 5.980 & 6.502 & 6.372 & 5.527 & 5.982 \\
\hline CAFFEIC-ACID & 1.500 & 1.470 & 1.275 & 1.380 & 1.502 & 1.472 & 1.277 & 1.382 \\
\hline CALCIUM & 450.0 & 441.0 & 382.5 & 414.0 & 450.002 & 441.002 & 382.502 & 414.002 \\
\hline CARBOHYDRATES & 12500 & 12250 & 10625 & 11500 & 12500 & 12250 & 10625 & 11500 \\
\hline CHOLINE & 300 & 294 & 255 & 276 & 300.002 & 294.002 & 255.002 & 276.002 \\
\hline COPPER & 0.0700 & 0.0686 & 0.0595 & 0.0644 & 0.0720 & 0.0706 & 0.0615 & 0.0664 \\
\hline FAT & 1700 & 1666 & 1445 & 1564 & 1700.002 & 1666.002 & 1445.002 & 1564.002 \\
\hline FIBER & 900 & 882 & 765 & 828 & 900.002 & 882.002 & 765.002 & 828.002 \\
\hline FLUORINE & 2.00 & 1.96 & 1.70 & 1.84 & 2.002 & 1.962 & 1.702 & 1.842 \\
\hline IODINE & 0.500 & 0.490 & 0.425 & 0.460 & 0.502 & 0.492 & 0.427 & 0.462 \\
\hline IRON & 0.850 & 0.833 & 0.723 & 0.782 & 0.852 & 0.835 & 0.725 & 0.784 \\
\hline KAEMPFEROL & 2.00 & 1.96 & 1.70 & 1.84 & 2.002 & 1.962 & 1.702 & 1.842 \\
\hline Magnesium & 0.400 & 0.392 & 0.340 & 0.368 & 0.402 & 0.394 & 0.342 & 0.370 \\
\hline NIAZIMIN & 3.00 & 2.94 & 2.55 & 2.76 & 3.002 & 2.942 & 2.552 & 2.762 \\
\hline Niacin (vitamin B3) & 0.800 & 0.784 & 0.680 & 0.736 & 0.802 & 0.786 & 0.682 & 0.738 \\
\hline OXALATE & 0 & 0 & 0 & 0 & 0 & 0 & 0 & 0 \\
\hline OXALIC-ACID & 0 & 0 & 0 & 0 & 0 & 0 & 0 & 0 \\
\hline PHOSPHORUS & 70.00 & 68.60 & 59.50 & 64.40 & 70.002 & 68.602 & 59.502 & 64.402 \\
\hline PROLAMINE & 86.800 & 85.064 & 73.780 & 79.856 & 86.802 & 85.066 & 73.782 & 79.858 \\
\hline PROTEIN & 6800 & 6664 & 5780 & 6256 & 6800.00 & 6664.00 & 5780.00 & 6256.00 \\
\hline Potassium & 255.00 & 249.90 & 216.75 & 234.60 & 255.002 & 249.902 & 216.752 & 234.602 \\
\hline $\begin{array}{l}\text { RIBOFLAVIN } \\
\text { (vitamin B2) }\end{array}$ & 0.050 & 0.049 & 0.043 & 0.046 & 0.052 & 0.051 & 0.045 & 0.048 \\
\hline Zinc & 0.1800 & 0.1764 & 0.1530 & 0.1656 & 0.1820 & 0.1784 & 0.1550 & 0.1676 \\
\hline TOCOPHEROLS & 29.00 & 28.42 & 24.65 & 26.68 & 29.002 & 28.422 & 24.652 & 26.682 \\
\hline Thiamin (vitamin B1) & 0.600 & 0.588 & 0.510 & 0.552 & 0.602 & 0.590 & 0.512 & 0.554 \\
\hline
\end{tabular}

All values are per 100 grams of edible portion; * The whole concentration values are mg.

Table 7. Analysis of the chemical components of Moringa roots cultivated under Egyptian conditions.

\begin{tabular}{lcccccccc}
\hline \multirow{2}{*}{\multicolumn{1}{c}{ Compound }} & \multicolumn{4}{c}{$\mathbf{2 0 1 2}$} & \multicolumn{5}{c}{$\mathbf{2 0 1 3}$} \\
\cline { 2 - 9 } & $\mathbf{A}_{\mathbf{1}}$ & $\mathbf{A}_{\mathbf{2}}$ & $\mathbf{A}_{\mathbf{3}}$ & $\mathbf{A}_{\mathbf{4}}$ & $\mathbf{A}_{\mathbf{1}}$ & $\mathbf{A}_{\mathbf{2}}$ & $\mathbf{A}_{\mathbf{3}}$ & $\mathbf{A}_{\mathbf{4}}$ \\
\hline $\begin{array}{l}\text { 1-BETA-D-GLUCOSYL-2,6- } \\
\text { DIMETHYL-BENZOATE } \\
\text { 4-(ALPHA-L- }\end{array}$ & 2.568 & 2.517 & 2.183 & 2.363 & 2.570 & 2.519 & 2.185 & 2.365 \\
$\begin{array}{l}\text { RHAMNOSYLOXY)- } \\
\text { BENZYLGLUCOCYANATE }\end{array}$ & 10.00 & 9.80 & 8.50 & 9.20 & 10.00 & 9.80 & 8.50 & 9.20 \\
$\begin{array}{l}\text { BENZYL- } \\
\text { ISOTHIOCYANATE }\end{array}$ & 6.998 & 6.858 & 5.948 & 6.438 & 7.000 & 6.860 & 5.950 & 6.440 \\
GLUCOTROPAEOLIN & 0.500 & 0.490 & 0.425 & 0.460 & 0.502 & 0.492 & 0.427 & 0.462 \\
PHYTOSTEROLS & 200.559 & 196.548 & 170.475 & 184.514 & 200.561 & 196.550 & 170.477 & 184.516 \\
PTERYGOSPERMIN & 185.569 & 181.858 & 157.734 & 170.724 & 185.571 & 181.860 & 157.736 & 170.726 \\
SPIROCHIN & 6.590 & 6.458 & 5.602 & 6.063 & 6.592 & 6.460 & 5.604 & 6.065 \\
ALKALOIDS & 1.000 & 0.980 & 0.850 & 0.920 & 1.002 & 0.982 & 0.852 & 0.922 \\
BENZYL-AMINE & 3.800 & 3.724 & 3.230 & 3.496 & 3.802 & 3.726 & 3.232 & 3.498 \\
MORINGINE & 5.200 & 5.096 & 4.420 & 4.784 & 5.202 & 5.098 & 4.422 & 4.786 \\
MORINGININE & 4.900 & 4.802 & 4.165 & 4.508 & 4.902 & 4.804 & 4.167 & 4.510 \\
\hline
\end{tabular}

All values are per 100 grams of edible portion. 
Table 8. Analysis of chemical constituents of Moringa seeds cultivated under Egyptian conditions.

\begin{tabular}{|c|c|c|c|c|c|c|c|c|}
\hline \multirow{2}{*}{ Compound } & \multicolumn{4}{|c|}{2012} & \multicolumn{4}{|c|}{2013} \\
\hline & $\overline{A_{1}}$ & $\mathbf{A}_{2}$ & $\overline{A_{3}}$ & $\overline{A_{4}}$ & $\overline{A_{1}}$ & $\overline{A_{2}}$ & $\overline{A_{3}}$ & $\mathbf{A}_{4}$ \\
\hline $\begin{array}{l}\text { 2,4-METHYLENE- } \\
\text { CHOLESTEROL }\end{array}$ & 11.00 & 10.78 & 9.35 & 10.12 & 11.00 & 10.78 & 9.35 & 10.12 \\
\hline 28-ISOAVENASTEROL & 5.00 & 4.90 & 4.25 & 4.60 & 5.00 & 4.90 & 4.25 & 4.60 \\
\hline $\begin{array}{l}\text { 4-(ALPHA-L- } \\
\text { RHAMNOSYLOXY)- } \\
\text { BENZYLGLUCOSINOLATE }\end{array}$ & 6.000 & 5.880 & 5.100 & 5.520 & 6.002 & 5.882 & 5.102 & 5.522 \\
\hline $\begin{array}{l}\text { 4-(ALPHA-L- } \\
\text { RHAMNOSYLOXY)- } \\
\text { BENZYLISOTHIOCYANATE }\end{array}$ & 90.000 & 88.200 & 76.500 & 82.800 & 90.002 & 88.202 & 76.502 & 82.802 \\
\hline ALPHA-TOCOPHEROL & 38.000 & 37.240 & 32.300 & 34.960 & 38.002 & 37.242 & 32.302 & 34.962 \\
\hline ARACHIDIC-ACID & 28.600 & 28.028 & 24.310 & 26.312 & 28.602 & 28.030 & 24.312 & 26.314 \\
\hline ASH & 32.000 & 31.360 & 27.200 & 29.440 & 32.002 & 31.362 & 27.202 & 29.442 \\
\hline BEHENIC-ACID & 33.200 & 32.536 & 28.220 & 30.544 & 33.202 & 32.538 & 28.222 & 30.546 \\
\hline BETA-CAROTENE & 0.100 & 0.000 & 0.000 & 0.000 & 0.000 & 0.000 & 0.000 & 0.000 \\
\hline BETA-SITOSTEROL & 0.800 & 0.784 & 0.680 & 0.736 & 0.802 & 0.786 & 0.682 & 0.738 \\
\hline BRASSICASTEROL & 0.9950 & 0.9751 & 0.8458 & 0.9154 & 0.9970 & 0.9771 & 0.8478 & 0.9174 \\
\hline CAMPESTANOL & 1.5560 & 1.5249 & 1.3226 & 1.4315 & 1.5580 & 1.5269 & 1.3246 & 1.4335 \\
\hline CAMPESTEROL & 2.590 & 2.538 & 2.202 & 2.383 & 2.592 & 2.540 & 2.204 & 2.385 \\
\hline CARBOHYDRATES & 207.29 & 203.14 & 176.20 & 190.71 & 207.29 & 203.15 & 176.20 & 190.71 \\
\hline CHOLESTEROL & 0.00 & 0.00 & 0.00 & 0.00 & 0.00 & 0.00 & 0.00 & 0.00 \\
\hline CLEROSTEROL & 0.500 & 0.490 & 0.425 & 0.460 & 0.502 & 0.492 & 0.427 & 0.462 \\
\hline DELTA-5-AVENASTEROL & 0.0050 & 0.0049 & 0.0043 & 0.0046 & 0.0070 & 0.0069 & 0.0063 & 0.0066 \\
\hline DELTA-7,14-STIGMASTANOL & 0.0520 & 0.0510 & 0.0442 & 0.0478 & 0.0540 & 0.0530 & 0.0462 & 0.0498 \\
\hline DELTA-7-AVENASTEROL & 0.0010 & 0.0010 & 0.0009 & 0.0009 & 0.0030 & 0.0030 & 0.0029 & 0.0029 \\
\hline DELTA-TOCOPHEROL & 22.000 & 21.560 & 18.700 & 20.240 & 22.002 & 21.562 & 18.702 & 20.242 \\
\hline EICOSANIC-ACID & 3.250 & 3.185 & 2.763 & 2.990 & 3.252 & 3.187 & 2.765 & 2.992 \\
\hline ERGOSTADIENOL & 8.259 & 8.094 & 7.020 & 7.598 & 8.261 & 8.096 & 7.022 & 7.600 \\
\hline FAT & 250.00 & 245.00 & 212.50 & 230.00 & 250.002 & 245.002 & 212.502 & 230.002 \\
\hline FIBER & 35.00 & 34.300 & 29.750 & 32.200 & 35.002 & 34.302 & 29.752 & 32.202 \\
\hline GADOLEIC-ACID & 4.800 & 4.704 & 4.080 & 4.416 & 4.802 & 4.706 & 4.082 & 4.418 \\
\hline GAMMA-TOCOPHEROL & 35.00 & 34.30 & 29.75 & 32.20 & 35.002 & 34.302 & 29.752 & 32.202 \\
\hline GLUCOSINOLATES & 70.00 & 68.60 & 59.50 & 64.40 & 70.002 & 68.602 & 59.502 & 64.402 \\
\hline LIGNOCERIC-ACID & 25.002 & 24.501 & 21.251 & 23.009 & 25.004 & 24.503 & 21.253 & 23.011 \\
\hline MYRISTIC-ACID & 7.500 & 7.350 & 6.375 & 6.900 & 7.502 & 7.352 & 6.377 & 6.902 \\
\hline OLEIC-ACID & 330.00 & 323.40 & 280.50 & 303.60 & 330.002 & 323.402 & 280.502 & 303.602 \\
\hline PALMITIC-ACID & 6.00 & 5.88 & 5.10 & 5.52 & 6.002 & 5.882 & 5.102 & 5.522 \\
\hline PROTEIN & 384.00 & 376.32 & 326.40 & 353.28 & 384.002 & 376.322 & 326.402 & 353.282 \\
\hline STEARIC-ACID & 56.00 & 54.88 & 47.6 & 51.52 & 56.002 & 54.882 & 47.602 & 51.522 \\
\hline STIGMASTANOL & 6.540 & 6.409 & 5.559 & 6.017 & 6.542 & 6.411 & 5.561 & 6.019 \\
\hline STIGMASTEROL & 2.5 & 2.45 & 2.125 & 2.30 & 2.502 & 2.452 & 2.127 & 2.302 \\
\hline TOCOPHEROLS & 44.00 & 43.12 & 37.40 & 40.48 & 44.002 & 43.122 & 37.402 & 40.482 \\
\hline WATER & 4.00 & 3.92 & 3.40 & 3.68 & 4.002 & 3.922 & 3.402 & 3.682 \\
\hline
\end{tabular}

All values are per 100 grams of edible portion. 
the best region, while the $\mathrm{A}_{3}$ showed the least response for chemical components in seeds (Bennett et al., 2003 and Berger et al., 1984).

Table (9) refers to the weight of the fresh leaves plant in places under study during the growing in two successive seasons of 2012 and 2013. The best region was A1, which gave 1667and1667.012 grams successively, while region A3 gave 1500.3 and 1500.312 grams successively, for fresh leaves weight. The best harvest period was after six months and twelve months, respectively. The obtained results explain that the environment of all regions was suitable for plant growth in respect to the high yield of Moringa leaves.

Table 9. Fresh weight per plant of fresh Moringa leaves cultivated under Egyptian conditions.

\begin{tabular}{lcccccccc}
\hline \multirow{2}{*}{ Compound } & \multicolumn{4}{c}{$\mathbf{2 0 1 2}$} & \multicolumn{3}{c}{$\mathbf{2 0 1 3}$} \\
\cline { 2 - 9 } & $\mathbf{A}_{\mathbf{1}}$ & $\mathbf{A}_{\mathbf{2}}$ & $\mathbf{A}_{\mathbf{3}}$ & $\mathbf{A}_{\mathbf{4}}$ & $\mathbf{A}_{\mathbf{1}}$ & $\mathbf{A}_{\mathbf{2}}$ & $\mathbf{A}_{\mathbf{3}}$ & $\mathbf{A}_{\mathbf{4}}$ \\
\hline After 2th months of Agriculture & 115.0 & 112.7 & 103.5 & 105.8 & 115.0 & 112.7 & 103.5 & 105.8 \\
After 4th months of Agriculture & 236.0 & 231.3 & 212.4 & 217.1 & 236.0 & 231.3 & 212.4 & 217.1 \\
After 6th months of Agriculture & 283.0 & 277.3 & 254.7 & 260.4 & 283.0 & 277.3 & 254.7 & 260.4 \\
After 8th months of Agriculture & 305.0 & 298.9 & 274.5 & 280.6 & 305.0 & 298.90 & 274.50 & 280.60 \\
After 10th months of Agriculture & 338.0 & 331.24 & 304.2 & 310.96 & 338.00 & 331.24 & 304.20 & 310.96 \\
After 12th months of Agriculture & 390.0 & 382.2 & 351.0 & 358.8 & 390.0 & 382.2 & 351.0 & 358.8 \\
Total weight of fresh Moringa & 1667.0 & 1633.7 & 1500.3 & 1533.6 & 1667.0 & 1633.7 & 1500.3 & 1533.7 \\
leaves (g) & 1663.7
\end{tabular}

All values for each gram of the weight of fresh Moringa leaves.

\section{RECOMMENDATIONS}

Moringa plants are cultivated in Egyptian soils whereas the Upper Egypt soil was better than Delta Egypt soil. In addition, it is advised to increase research and studies for this plant because of its nutritional and economic values, specially facing poverty and the shortage of proteins source for Egyptian people.

\section{REFERENCES}

A.A.C.C. (2000). Approved Methods of American Association of Cereal Chemists. The $10^{\text {th }}$ Ed. St. Paul. Minnesota, USA.

Abrams, B.; Duncan, D. and Hertz-Piccioto, I. (1993). A prospective study of dietary intake and acquired immune deficiency syndrome in HIV-sero-positive homosexual men. Journal of Acquired Immune Deficiency Syndrome, 8:949958.
Abuye, C.; Omwega, A.M. and Imungi, J.K. (1999). Familial tendency and dietary association of goitre in Gamo-Gofa, Ethiopia. East African Medical Journal, 76:447-451.

Akhtar A. H. and Ahmad, K.U. (1995). Antiulcerogenic evaluation of the methanolic extracts of some indigenous medicinal plants of Pakistan in aspirin-ulcerated rats. Journal of Ethnopharmacology, 46:1-6.

Akowuah, G.A.; Ismail, Z.; Norhayati, I. and Sadikun, A. (2005). The effects of different extraction solvents of varying polarities of polyphenols of Orthosiphon stamineus and evaluation of the free radical-scavenging activity. Food Chemistry, 93:311-317.

Al-Saikhan, M.S.; Howard, L.R. and Miller, J.C. (1995). Antioxidant activity and total phenolics in different genotypes of potato 


\section{E.A.E. El Ghadban et al.}

(Solanum tuberosum L.) J. Food Sci., 60(2):341-343.

Anderson, D.M.W., Bell, P.C.; Gill, M.C.L.; McDougall, F.J. and McNab, C.G.A. (1986). The gum exudates from Chloroxylon swietenia, Sclerocarya caffra, Azadirachta indica and Moringa oleifera. Phytochemistry, 25(1):247-249.

Anwar, F. and Bhanger, M.I. (2003). Analytical characterization of Moringa oleifera seed oil grown in temperate regions of Pakistan. Journal of Agricultural and Food Chemistry, 51:6558-6563.

A.O.A.C. (2003). Official Methods of Analysis of the Association of Official's Analytical Chemists. The $17^{\text {th }}$ Ed., Arlington, Virginia.

A.O.C.S. (1998). Official methods and recommended practices of the American Oil Chemist's Society, 5th ed. Champaign, USA.

Asres, K. (1995). The major constituents of the acetone fraction of Ethiopian Moringa stenopetala leaves. Mansoura Journal of Pharmacological Science, 11(1):55-64.

Babu, S.C. (2000). Rural nutrition interventions with indigenous plant foods: a case study of vitamin deficiency in Malawi. International Food Policy Research Institute, Washington, DC. Biotechnology, Agronomy Soc. Environ., 4(3):169-179.URL: http://www.bib.fsagx .ac.be/library/base/text/v4n3/169.pdf.

Badgett, B.L. (1964). Part I. The Mustard Oil Glucoside from Moringa oleifera Seed. Ph.D. Thesis, Rice University (student of Martin G. Ettlinger), Houston, TX, USA.

Barminas, J.T.; Charles, M. and Emmanuel, D. (1998). Mineral composition of nonconventional leafy vegetables. Plant Foods for Human Nutrition Dordrecht, 53(1):29-36.

Bennett, R.N.; Mellon, F.A.; Foidl, N.; Pratt, J.H.; DuPont, M.S.; Perkins, L. and
Kroon, P.A. (2003). Profiling glucosinolates and phenolics in vegetative and reproductive tissues of the multi-purpose trees Moringa oleifera L. (Horseradish tree) and Moringa stenopetala L. Journal of Agricultural and Food Chemistry, 51:3546-3553.

Berger, M.R.; Habs, M.; Jahn, S.A. and Schmahl, S. (1984). Toxicological assessment of seeds from Moringa oleifera and Moringa stenopetala, two highly efficient primary coagulants for domestic water treatment of tropical raw waters. East African Medical Journal, 61: 712-716.

Bharali, R.; Tabassum, J. and Azad, M.R.H. (2003). Chemomodulatory effect of Moringa oleifera, Lam, on hepatic carcinogen metabolizing enzymes, antioxidant parameters and skin papillomagenesis in mice. Asian Pacific Journal of Cancer Prevention, 4:131-139.

Caceres, A. and Lopez, S. (1991). Pharmacological properties of Moringa oleifera: 3. Effect of seed extracts in the treatment of experimental pyodermia. Fitoterapia, 62(5):449-450.

Caceres, A.; Cabrera, O.; Morales, O.; Mollinedo, P. and Mendia, P. (1991). Pharmacological properties of Moringa oleifera: 1. Preliminary screening for antimicrobial activity. Journal of Ethnopharmacology, 33:213-216.

Caceres, A.; Saravia, A.; Rizzo, S.; Zabala, L.; De.Leon, E. and Nave, F. (1992). Pharmacologic properties of Moringa oleifera: 2. Screening for antispasmodic, anti-inflammatory and diuretic activity. Journal of Ethnopharmacology, 36: 233237.

Chawla, S.; Saxena, A. and Seshadri, S. (1988). In-vitro availability of iron in various green leafy vegetables. Journal of the Science of Food and Agriculture, 46(1):125-128.

Costa-Lotufo, L.V.; Khan, M.T.H.; Ather, A.; Wilke, D.V.; Jimenez, P.C.; Pessoa, 
C.; de Moraes, M.E.A. and de Moraes, M.O. (2005). Studies of the anticancer potential of plants used in Bangladeshi folk medicine. Journal of Ethnopharmacology, 99:21-30.

D'Souza, J. and Kulkarni, A.R. (1993). Comparative studies on nutritive values of tender foliage of seedlings and mature plants of Moringa oleifera Lam. Journal of Economic and Taxonomic Botany, 17(2):479-485.

Dahot, M.U. (1998). Antimicrobial activity of small protein of Moringa oleifera leaves. Journal of the Islamic Academy of Sciences, 11(1):6.
Singleton, V.L.; Orthofer, R. and LamuelaRavento, R.M. (1999). Analysis of total phenols and other oxidation substrates and antioxidants by means of folinciocalteu reagent. Methods in Enzymology, 299:152-178.

Taga, M.S.; Miller, E.E. and Pratt, D.E. (1984). Chia seeds as a source of natural lipids antioxidants. J. Am. Oil Chem. Soc., 61:928-993.

Wikipedia (2010): www.wikipedia.com.

$$
\begin{aligned}
& \text { دراسات على المكونات الكيميائية لنبات المورينجا اوليفيرا النامية تحت الظروف المصرية }
\end{aligned}
$$

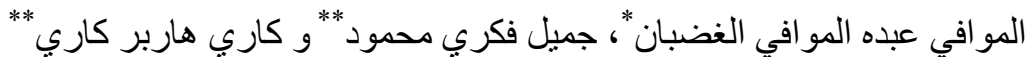

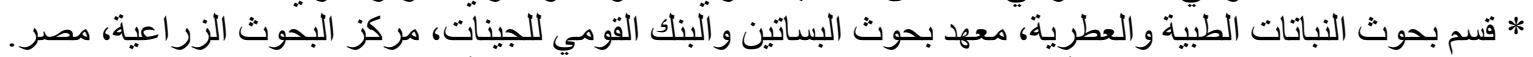

$$
\begin{aligned}
& \text { * * قم الأغذية الوظيفية، المعهد القومي للتغذية الكندية، أوتوا، كندا. }
\end{aligned}
$$

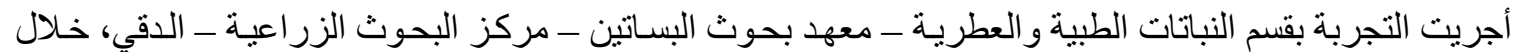

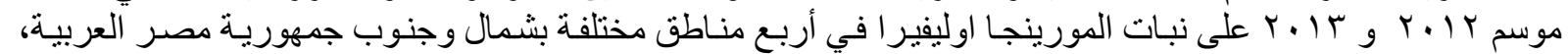

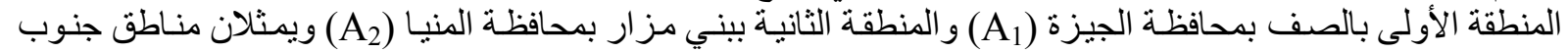

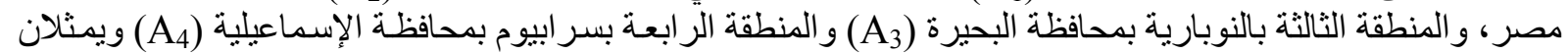

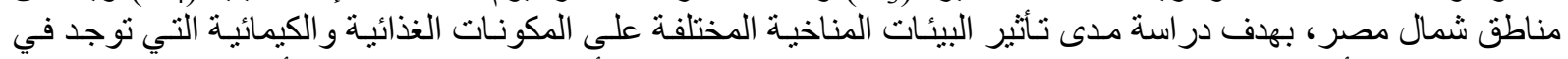

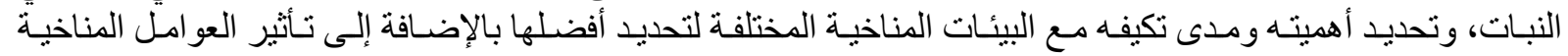

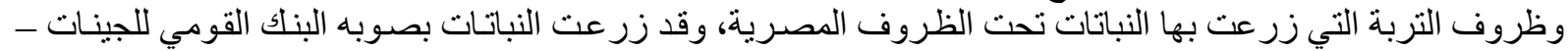

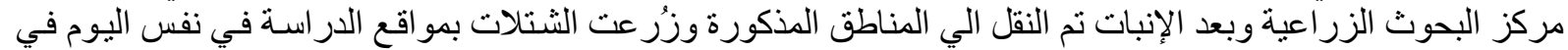

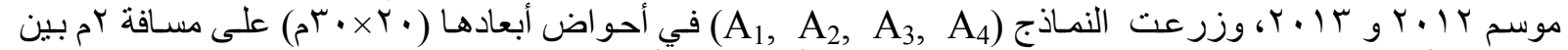

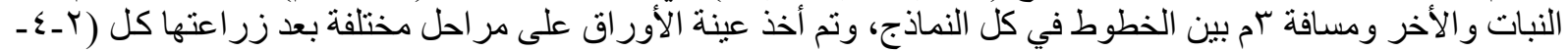

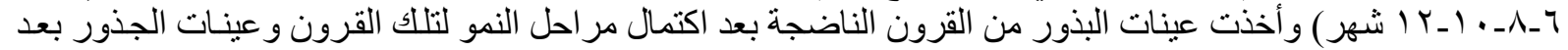

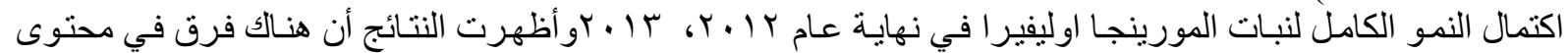

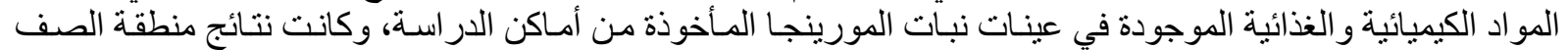

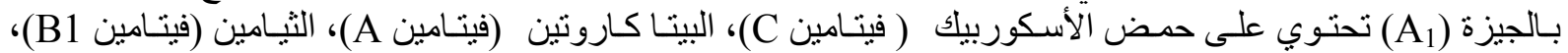

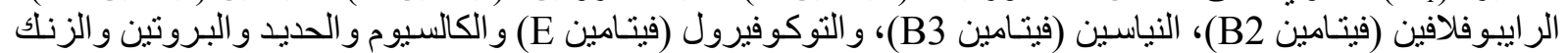

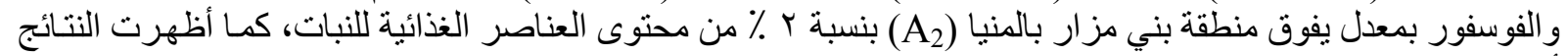

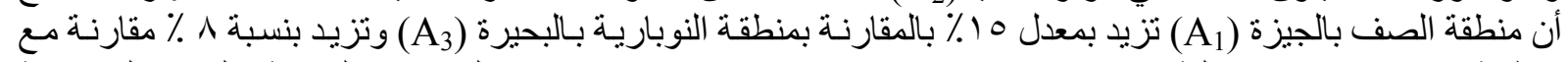

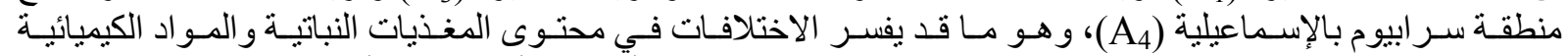

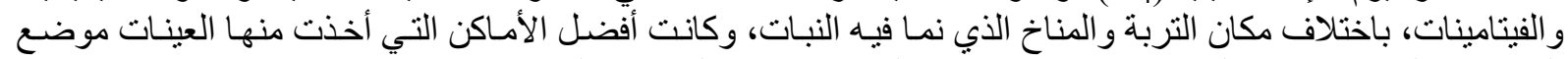

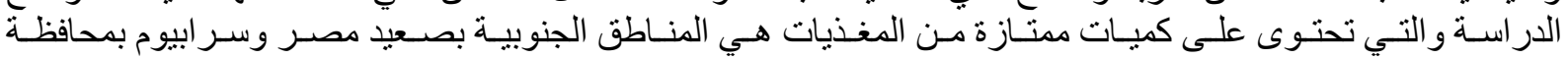


